\title{
AVALIAÇÃO DA FUNÇÃO COGNITIVA DE IDOSOS APÓS A REALIZAÇÃO DE UM PROGRAMA DE EXERCÍCIOS FÍSICOS
}

Vinícius Gustavo Gimenes Turato; Juliana Rosini da Silva; Letícia Costa Ribeiro; Regina Celi Trindade Camargo; Claudia Regina Sgobbi Faria

Faculdade de Ciências e Tecnologia FCT/UNESP - Campus de Presidente Prudente - SP. E-mail: vinicius_gimenes3@hotmail.com

\section{RESUMO}

Segundo informações do último censo, o número de idosos representa 10,8\% da população brasileira. Com o aumento da expectativa de vida, o Brasil será o sexto país do mundo com o maior número de pessoas idosas até 2025. O envelhecimento refer-se a um processo altamente complexo e variável, progressivo, sendo influenciado por vários fatores, como a execução de exercícios físicos; além de, sofrer perdas inevitáveis, como o declínio da função cognitiva. Avaliar função cognitiva de idosos após um programa de fisioterapia com enfoque em exercícios físicos regulares e supervisionados. Quarenta e três idosos foram avaliados quanto à função cognitiva após doze meses de um programa de fisioterapia. Nível de significância considerado foi $5 \%$. Os resultados do MEEM, antes e após doze meses de intervenção não apresentaram diferença estatística significante. O exercício físico pode ser eficaz na manutenção da função cognitiva de idosos.

Palavras-chave: Idosos, Exercício Físico e Função Cognitiva.

\section{INTRODUÇÃO E OBJETIVO}

Segundo informações do último censo, o número de idosos é de, aproximadamente, 20.590.599, representando $10,8 \%$ da população brasileira (IBGE, 2011) ${ }^{1}$. Esses dados estão associados à diminuição das taxas de natalidade e mortalidade, resultando em aumento significativo da proporção de idosos ${ }^{2}$. Com o aumento da expectativa de vida, segundo a OMS, o Brasil será o sexto país do mundo com o maior número de pessoas idosas até $2025^{3}$.

O processo de envelhecer refere-se a um fenômeno altamente complexo e variável, progressivo, envolvendo mecanismos deletérios que afetam a capacidade de desempenhar um grande número de funções. Trata-se de um processo multidimensional e multidirecional, pois há uma variabilidade na taxa e direção das mudanças (ganhos e perdas) em diferentes características de cada indivíduo e entre indivíduos ${ }^{4}$.

Com o avanço da idade, deterioração estrutural e funcional ocorre na maioria dos sistemas fisiológicos, mesmo na ausência de doenças. Estas alterações fisiológicas relacionadas à idade comprometem vários tecidos, sistemas de órgãos e funções que, cumulativamente, podem interferir nas atividades de vida diária ${ }^{5}$. 
Também, ao envelhecer, pode ocorrer redução na função cognitiva em pessoas acima de 60 anos de idade. Esta constatação subjetiva é bastante comum, uma vez que os idosos, em geral, se queixam de dificuldades com a memória e outras habilidades cognitivas, particularmente quando comparam o desempenho atual com o do passado ${ }^{6,7}$.

Uma das explicações mais aceita para a compreensão dos fatores que estariam relacionados com as perdas cognitivas está associado à redução da função cardiovascular decorrente do envelhecimento. Esta redução levaria a um decréscimo progressivo na oxigenação e uma hipóxia tecidual ao longo do tempo, por fim implicando em declínio cognitivo ${ }^{8}$.

No entanto, a OMS afirma que a prática regular de exercícios pode prevenir, minimizar e/ou reverter muitos dos problemas que freqüentemente acompanham o processo de envelhecimento ${ }^{9}$.

Há evidências na literatura de que o exercício físico pode influenciar de forma positiva a função cognitiva de idosos. Estudos observaram que um estilo de vida ativo diminui os efeitos deletérios do declínio cognitivo ${ }^{7}$.

Em estudos de revisão, Antunes et al. (2006) e Rolland et al. (2007) encontraram vários achados que evidenciaram melhora das funções cognitivas de pessoas idosas, com a prática de exercícios $^{8,10}$.

Considerando-se, então, que a população de idosos está aumentando e, que o declínio da função cognitiva é conseqüência natural do processo de envelhecimento, este estudo teve por objetivo avaliar os efeitos do exercício físico sobre a função cognitiva de idosos após um programa de fisioterapia com enfoque em exercícios físicos regulares e supervisionados.

\section{METODOLOGIA}

Estudo retrospectivo, que contou com a participação de quarenta e três indivíduos, idade entre 60 e 90 anos, integrantes do programa fisioterapêutico preventivo aos efeitos deletérios da terceira idade da FCT/UNESP. Após esclarecimentos dos objetivos e métodos da pesquisa, os participantes assinaram um termo de consentimento livre e esclarecido. Cabe ressaltar que a pesquisa é parte do estudo intitulado "EFEITOS DO EXERCÍCIO FÍSICO SOBRE O PROCESSO DE ENVELHECIMENTO", sob responsabilidade da Prof(a) Dr(a) Cláudia Regina Sgobbi de Faria e a Pro(a) Ms(a) Regina Celi Trindade Camargo, que foi aprovado pelo Comitê de Ética em Pesquisa da própria instituição (protocolo 51910). 
O programa é fundamentado na prática de exercícios físicos regulares e supervisionados, visando promover manutenção e/ou benefícios em habilidades como: força, resistência, flexibilidade, equilíbrio, coordenação, cognição, etc. Este apresenta freqüência semanal de três sessões, com duração de uma hora cada.

Os idosos inclusos apresentavam vínculo de pelo menos seis meses com o programa e foram avaliados em dois momentos, antes e após doze meses de intervenção.

A função cognitiva foi avaliada pelo Mini-Exame do Estado Mental (MEEM). O MEEM é um instrumento composto por 30 itens, agrupados em 7 categorias: orientação temporal, orientação espacial, registro de três palavras, atenção e cálculo, recordação das três palavras, linguagem e capacidade construtiva visual. Sendo que, o resultado do MEEM varia de 0 a 30 pontos ${ }^{11}$. Todavia, como este sofre influência da escolaridade, Brucki et al. (2003) analisaram uma amostra brasileira e sugeriram os seguintes valores para estudos em nosso país: analfabetos $=20$ pontos; de 1 a 4 anos de escolaridade $=25$ pontos; de 5 a 8 anos de escolaridade $=26$ pontos; de 9 a 11 anos de escolaridade $=28$ pontos; e para indivíduos com escolaridade superior a 11 anos $=29$ pontos ${ }^{12}$.

\section{RESULTADOS}

Tabela 1. Caracterização da amostra quanto a idade e o IMC, expressos em valores de média e desvio-padrão.

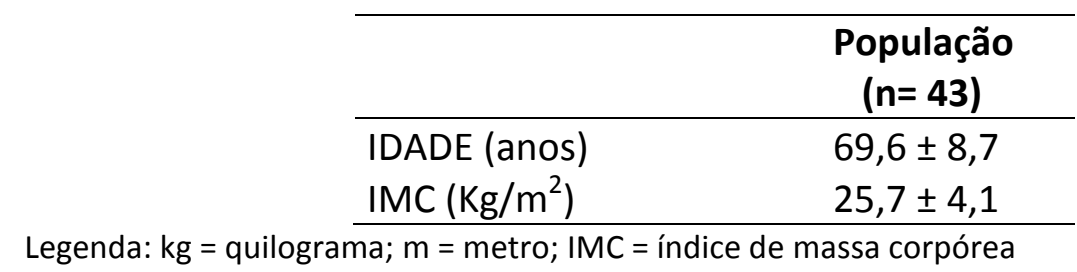

Como mencionado anteriormente, o nível de escolaridade possui relação direta com o resultado do MEEM, sendo assim, na Figura 1, os indivíduos foram alocados em diferentes graus de ensino, de acordo com seus níveis de formação. 


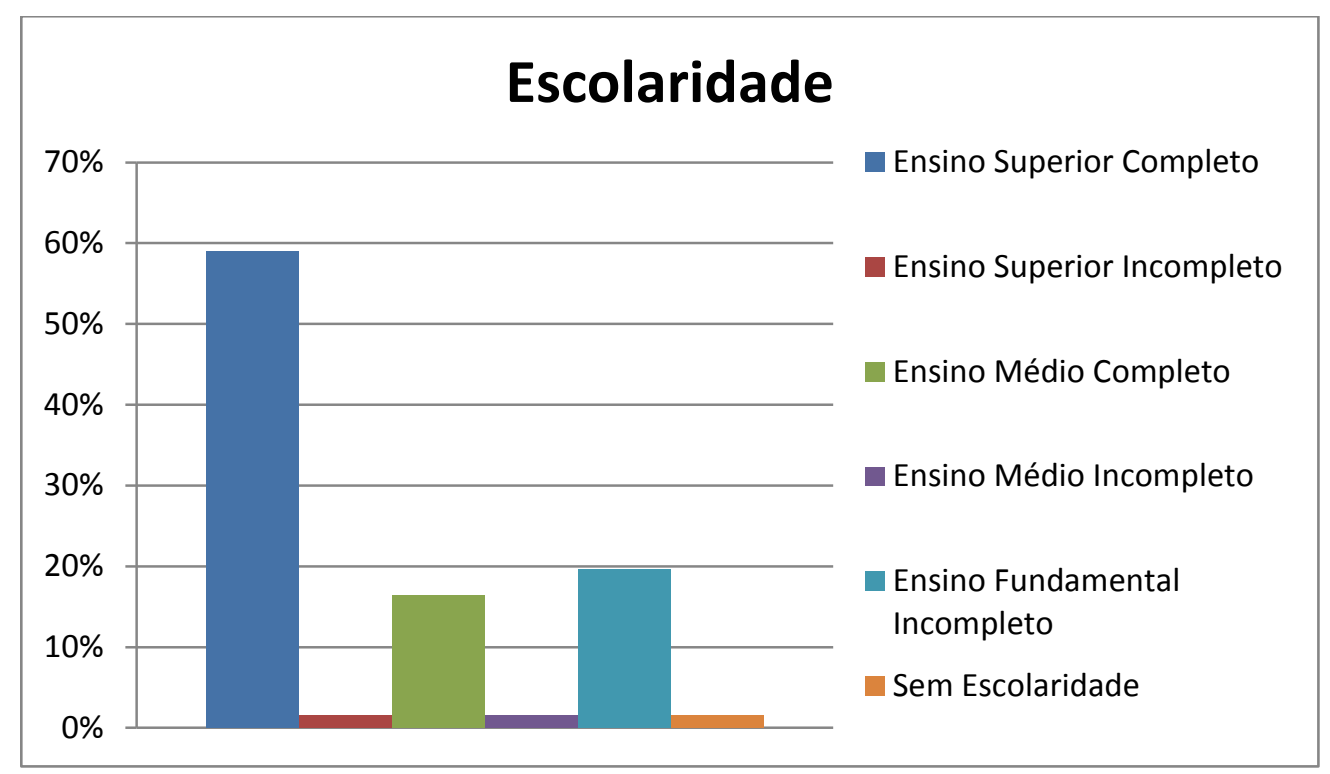

Figura 1. Representação gráfica, em valores percentuais, dos indivíduos de acordo com o grau de escolaridade.

Pode-se observar que, a maioria dos participantes (59\%) concluiu o ensino superior, totalizando, aproximadamente, quinze anos de ensino formal. Esta informação é muito interessante, pois demonstra um alto nível de escolaridade dos indivíduos, o que não é relatado na literatura, sendo que, a maioria dos trabalhos apresenta uma média de estudo de quatro a sete anos.

Os valores dos resultados do MEEM, antes $(27,3 \pm 2,8)$ e após $(27,2 \pm 2,7)$ doze meses de intervenção, estão apresentados na Figura 2. Não houve diferença estatística significante entre os momentos.

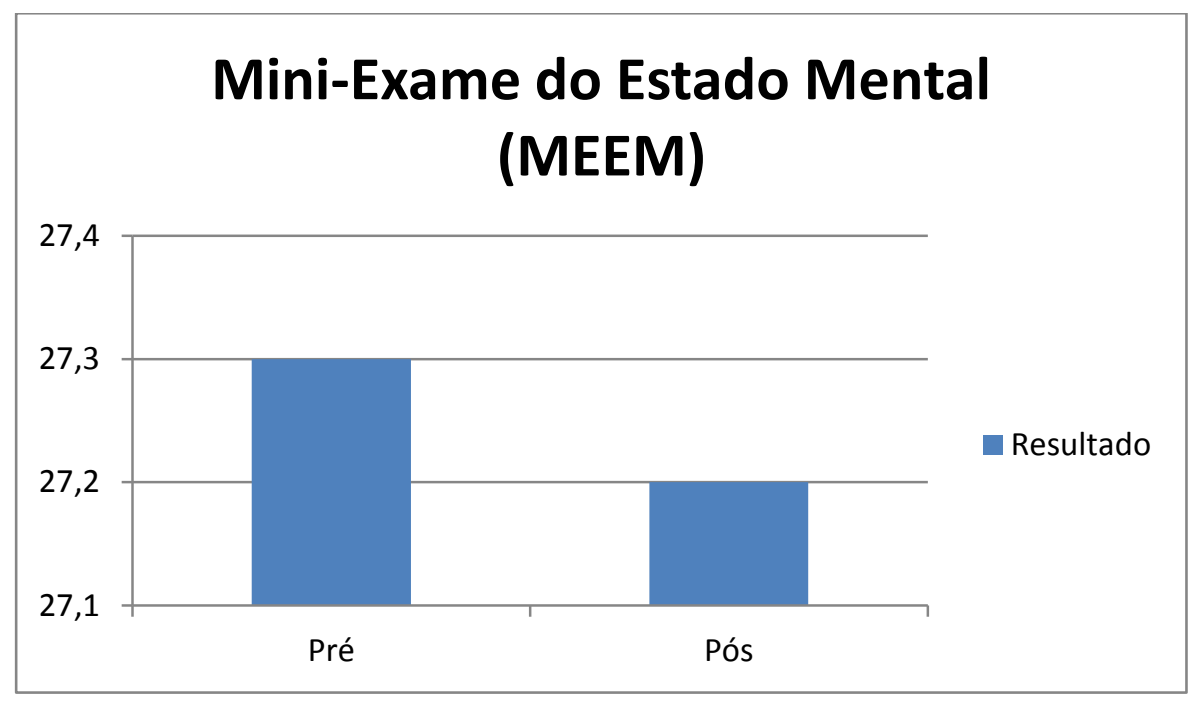

Figura 2. Representação gráfica dos resultados (média) do MEEM em ambos os momentos de avaliação. 


\section{DISCUSSÃO}

No presente estudo não foi observada diferença estatística significante entre os momentos de avaliação. Dessa forma, houve uma manutenção do resultado do MEEM após doze meses de prática de exercício físico, sugerindo uma preservação da função cognitiva no decorrer desse período.

Estudos realizados anteriormente demonstraram que a prática de exercício físico possui efeitos benéficos sobre a função cognitiva de indivíduos idosos.

Hernandez et al. (2010) verificaram que houve manutenção das funções cognitivas de idosos portadores de doença de Alzheimer após a realização de um programa de exercícios físicos quando comparados ao grupo que não participou do protocolo, que apresentaram declínio significativo dessa variável ${ }^{11}$.

Além disso, Borges et al. (2009) concluíram que o exercício físico está associado a melhora de déficits cognitivos para idosos que participaram assiduamente de um protocolo de exercícios físicos ${ }^{13}$.

Contudo, em estudo realizado por Banhato et al. (2009), apesar de os idosos ativos terem obtido resultados significantemente mais altos em relação aos idosos sedentários, quando o efeito da escolaridade foi controlado não se verificou diferença entre os grupos ${ }^{9}$.

Sabe-se que, segundo a literatura, a escolarização está associada à cognição, uma vez que quanto menor é a escolaridade, pior é o desempenho cognitivo ${ }^{9}$. Todavia, apesar do não controle desta variável no presente estudo, esta não deve ter contribuído diretamente no resultado, tendo em vista que foi realizada uma comparação intragrupo em momentos divergentes.

A prática de exercício físico como alternativa para melhorar a função cognitiva mostra-se relevante, especialmente por sua aplicabilidade, pois se trata de um método relativamente de baixo custo, que pode ser apresentado a grande parte da população. Contudo, deve-se avaliar sobre o exercício não somente como uma alternativa não medicamentosa, mas também como um coadjuvante ${ }^{8}$.

Houve dificuldade no estudo, quanto ao fato de o MEEM ser um instrumento global da função cognitiva, podendo não possuir sensibilidade adequada para mensurar a influencia do exercício. Outra limitação está relacionada à ausência de um grupo controle, o que não permite afirmar categoricamente que os resultados encontrados sejam em decorrência da intervenção. 


\section{CONCLUSÃO}

Diante dos resultados obtidos, conclui-se que o programa de fisioterapia com enfoque na prática de exercícios físicos regulares e supervisionados pode ser eficaz na manutenção da função cognitiva de idosos.

\section{REFERÊNCIAS}

1. Meurer ST, Borges $\amalg$, Benedetti TRB, Mazo GZ. ASSOCIAÇÃO ENTRE SINTOMAS DEPRESSIVOS, MOTIVAÇÃO E AUTOESTIMA DE IDOSOS PRATICANTES DE EXERCÍCIOS FÍSICOS. Revista Brasileira de Ciências do Esporte. 2012 Jul/Set;34(3):683-695.

2. Maués CR, Paschoal SMP, Jaluul O, França CC, Filho WJ. Avaliação da qualidade de vida: comparação entre idosos jovens e muito idosos. Revista Brasileira Clínica Médica. 2010 Set/Out;8(5):405-410.

3. Silva MFD, Goulart NBA, Lanferdini FJ, Marcon M, Dias CP. Relação entre os níveis de atividade física e qualidade de vida de idosos sedentários e fisicamente ativos. Rev Bras Geriatr Gerontol. 2012 Out/Dez;15(4):634-642.

4. Franchi KMB, Montenegro RM. Atividade física: uma necessidade para boa saúde na terceira idade. Rev $\quad$ Bras 2005;18(3):152-6. http://dx.doi.org/10.5020/18061230.2005.p152

5. Chodzko-Zajko WJ, Proctor DN, Fiatarone Singh, MA, Minson CT, Nigg CR, Salem GJ, Skinner JS. Exercise and physical activity for older adults. Medicine and Science in Sports and Exercise. 2009 Jul; 41:1510-1530. http://dx.doi.org/10.1249/MSS.0b013e3181a0c95c

6. Leite, MT, Winck, MT, Hildebrandt, LM, Kirchner, RM, Silva, LAAD. Quality of life and cognitive level of elderly members of conviviality groups. Revista Brasileira de Geriatria e Gerontologia. 2012 Jul/Sep;15(3):481-492. http://dx.doi.org/10.1590/S1809-98232012000300009

7. Chiari, H, Mello, MT, Rezeak, P, Antunes, HKM. Exercício físico, atividade física e os benefícios sobre a memória de idosos. Revista Psicologia e Saúde. 2010 Jan/Jun; 2(1):42-49.

8. Antunes, HK, Santos, RF, Cassilhas, R, Santos, RV, Bueno, OF, Mello, MTD. Exercício físico e função cognitiva: uma revisão. Revista Brasileira de Medicina do Esporte. 2006 Mar/Apr;12(2):108114. http://dx.doi.org/10.1590/S1517-86922006000200011

9. Banhato, EF, Scoralick, NN, Guedes, DV, Atalaia-Silva, KC, Mota, MM. Atividade física, cognição e envelhecimento: estudo de uma comunidade urbana. Psicologia: teoria e prática. 2009 Jun;11(1):76-84.

10. Rolland Y, Pillard F, Klapouszczak A, et al. Exercise Program for Nursing Home Residents with Alzheimer's Disease: A 1-Year Randomized, Controlled Trial. J Am Geriatr Soc 2007 Feb;55(2):15865. http://dx.doi.org/10.1111/j.1532-5415.2007.01035.x 
11. Hernandez, SSS, Coelho, FGM, Gobbi, S, Stella, F. Efeitos de um programa de atividade física nas funções cognitivas, equilíbrio e risco de quedas em idosos com demência de Alzheimer. Rev Bras Fisioter, 2010 Jan/Feb;14(1):68-74. http://dx.doi.org/10.1590/S1413-35552010000100011

12. Brucki SMD, Nitrini R, Caramelli P, Bertolucci PHF, Okamoto IH. Sugestões para o uso do miniexame do estado mental no Brasil. Arq Neuropsiquiatr. 2003;61(3B):777-81.

http://dx.doi.org/10.1590/S0004-282X2003000500014

13. Borges, L, Benedetti, TRB, Mazo, GZ. Exercício físico, déficits cognitivos e aptidão funcional de idosos usuários dos centros de saúde de Florianópolis. Revista Brasileira de Atividade Física \& Saúde. 2009 Apr;13(3):167-177. 